\title{
Turbulent Magnetic Field Amplification behind Strong Shock Waves in GRB and SNR
}

\author{
Tsuyoshi Inoue \\ Department of Physics and Mathmatics, Aoyama Gakuin University, \\ Fuchinobe, Chuou-ku, Sagamihara 252-5258, Japan \\ email: inouety@phys.aoyama.ac.jp
}

\begin{abstract}
Using three-dimensional (special relativistic) magnetohydrodynamics simulations, the amplification of magnetic field behind strong shock wave is studied. In supernova remnants and gamma-ray bursts, strong shock waves propagate through an inhomogeneous density field. When the shock wave hit a density bump or density dent, the Richtmyer-Meshkov instability is induced that cause a deformation of the shock front. The deformed shock leaves vorticity behind the shock wave that amplifies the magnetic field due to the stretching of field lines.
\end{abstract}

Keywords. magnetic fields, turbulence, gamma rays: bursts, supernova remnants

\section{Motivation and Results of Simulations}

It is well known that magnetic fields play a very important role in high-energy astrophysical phenomena through particle acceleration and synchrotron emission. Strong magnetic fields around shock waves are often required to explain the observed emission. For example, at the shocks in supernova remnants (SNRs) and gamma-ray bursts (GRBs), magnetic field strengths orders of magnitude larger than their ambient fields are needed.

In this paper, we show that the magnetic field can be amplified far beyond the shock compression value behind the shock wave, if the shock wave propagates through an inhomogeneous density field (Giacalone \& Jokipii 2007, Inoue et al. 2009, 2010, 2011, 2012). In the case of the SNR, observations have shown that the young SNR RX J1713.73946 is interacting with molecular clouds (e.g., Fukui et al. 2012) where there is strong evidence of magnetic field amplification up to $B \sim 1 \mathrm{mG}$ (Uchiyama et al. 2007).

The left panel of Fig. 1 shows the result of the three-dimensional (3D) magnetohydrodynamics (MHD) simulation of the shock propagation in a realistic cloudy ISM with $B_{\text {ini }}=5.0 \mu \mathrm{G}$ (Inoue et al. 2012, see also Inoue \& Inutsuka 2008, 2009 for the cloud formation). Because the interaction between the shock wave and cloud generates vorticity behind the shock through the induction of the Richtmyer-Meshkov instability, the shocked ISM that corresponds to the SNR becomes highly turbulent. The turbulence behind the shock wave amplifies magnetic field by stretching field lines. In the present case, the maximum field strength reaches $B_{\max } \sim 1 \mathrm{mG}$ (corresponding plasma $\beta \sim 1$ ) and the average field strength becomes $\langle|B|\rangle \sim 100 \mu \mathrm{G}$. The strongly magnetized regions with $B \sim 1 \mathrm{mG}$ are located at the interface regions of the clouds where strong shear flows are developed by the shock-cloud interaction. Their spatial scale, $\sim 0.05 \mathrm{pc}$, which is essentially determined by the thickness of the interface due to the thermal conduction (Inoue et al. 2006, 2007), agrees pretty well with the scale of the observed amplified regions of $B \sim 1 \mathrm{mG}$ (Uchiyama et al. 2007). 

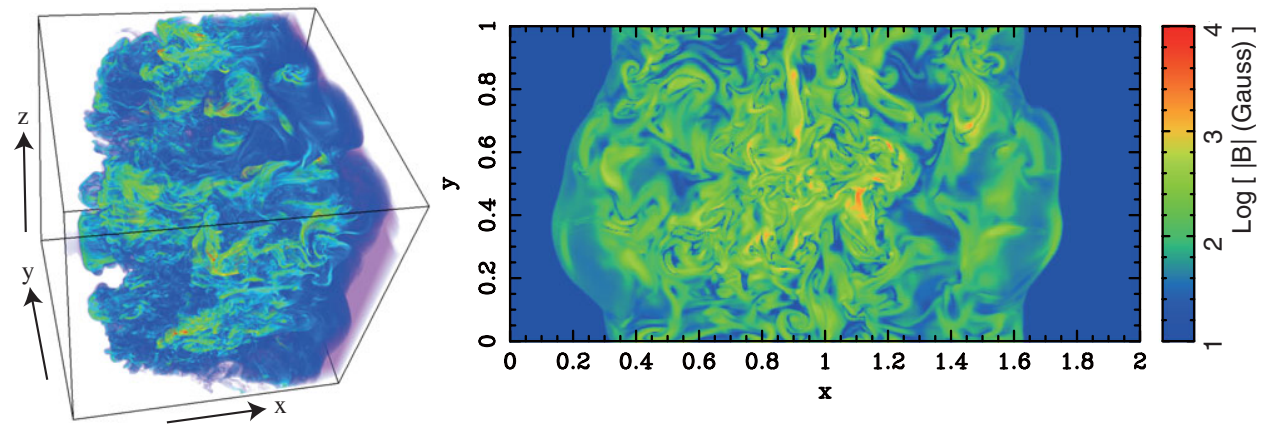

Figure 1. Left: map of magnetic field strength as a result of the MHD simulation of the shock propagation in inhomogeneous cloudy ISM. Regions in blue, green and red represent the regions with $B \lesssim 100 \mu \mathrm{G}, B \gtrsim 100 \mu \mathrm{G}$, and $B \gtrsim 500 \mu \mathrm{G}$, respectively. Right: $2 \mathrm{D}$ slice of magnetic field strength as a result of the $3 \mathrm{D}$ relativistic MHD simulation of inhomogeneous flow collision.

The GRBs are believed to be caused by the collisions of relativistic jets that are released when a black hole is formed. Because the jets are released very intermittently with time, it is reasonable to expect the inhomogeneous structure of the jets. Right panel of Fig. 1 shows the result of the 3D special relativistic MHD simulation of the relativistic jet-jet collision (Inoue et al. 2011). Owing to the density fluctuations imprinted in the colliding flows, the postshock layer becomes highly turbulent and the turbulence amplifies the magnetic field. We find that the velocity dispersion of turbulence induced behind the shock is proportional to the upstream density dispersion $(\Delta v \sim \Delta \rho)$ and the velocity dispersion saturates when it reaches the postshock sound speed $(\sim c / \sqrt{3})$ at $\Delta \rho \sim \rho_{0}$. The magnetic field behind the shock is amplified until the magnetic energy becomes comparable to the kinetic energy of the decaying turbulence that is powered only at the shock front.

\section{Acknowledgements}

I would like to thank A. MacFadyen and J. Mao for fruitful discussions. Numerical computations were carried out on XT4 at the Center for Computational Astrophysics (CfCA) of National Astronomical Observatory of Japan. This work is supported by Grant-in-aids from the Ministry of Education, Culture, Sports, Science, and Technology (MEXT) of Japan, No.22·3369 and 23740154 .

\section{References}

Fukui, Y. et al. 2012, ApJ, 746, 82

Giacalone, J. \& Jokipii, J. R. 2007, ApJ, 663, L41

Inoue, T., Inutsuka, S., \& Koyama, H. 2006, ApJ, 652, 1331

Inoue, T., Inutsuka, S., \& Koyama, H. 2007, ApJ, 658, L99

Inoue, T. \& Inutsuka, S. 2008, $A p J, 687,303$

Inoue, T. \& Inutsuka, S. 2009, ApJ, 704, 161

Inoue, T., Yamazaki, R., \& Inutsuka, S. 2009, ApJ, 695, 825

Inoue, T., Yamazaki, R., \& Inutsuka, S. 2010, ApJ, 723, L108

Inoue, T., Asano, K., \& Ioka, K. 2011, ApJ, 734, 77

Inoue, T., Yamazaki, R., Inutsuka, S., \& Fukui, Y. 2012, ApJ, 744, 71

Uchiyama, Y. et al. 2007, Nature, 449, 576 